\title{
Search times for combinations of visual, phonemic, and semantic targets in reading prose ${ }^{1}$
}

\author{
GILLIAN COHEN ${ }^{2}$ \\ INSTITUTE OF EXPERIMENTAL PSYCHOLOGY, OXFORD
}

\begin{abstract}
Subjects read passages of prose, canceling targets that were visual (letters), acoustic (phonemes), or semantic (words of a specified category). Each of the targets occurred singly and in all possible double and triple combinations. Comparison of the search times reveals that semantic processing adds little or no extra time when combined with acoustic or with visual and acoustic searches, i.e., it occurs in parallel. Other combinations of processing yield search times that are slightly faster than a serial model would predict and are best interpreted as reflecting an overlapping sequence of operations.
\end{abstract}

In reading, three kinds of processing may take place: a visual processing resulting in recognition of letter shapes, an acoustic processing resulting in phoneme recognition, and a semantic processing resulting in recognition of meaning. A previous study (Cohen, 1968) suggested that these levels of processing are arranged hierarchically. The question then arises as to whether any of these three types of processing can be carried out in parallel, or whether analysis must always progress serially from one stage to the next.

Neisser (1964) found that several targets could be searched for simultaneously as fast as a single target, although Kaplan et al (1966) reported a linear increase in search times as the number of targets increased. Whatever the reason for the divergence of these results, they refer only to search for multiple targets of the same type. So, whether or not same-level analyzers can operate in parallel, it remains an entirely separate question as to whether analyses of different types, at different levels, are parallel or serial. Moreover, the question is not dichotomous. In the reading of continuous passages of prose, a third possibility, intermediate between a strictly parallel and a strictly serial model, presents itself. It is quite possible that the different levels of processing occur in an overlapping sequence, so that, for example, the output of lower-level visual shape analysis is beginning to be processed at a semantic level while further visual analysis is still ongoing. Obviously, some visual processing must precede both semantic and acoustic decisions, so that visual analysis could not occur completely in parallel with either phonemic or semantic analysis. But it is logically quite possible that these combined analyses take place in the overlapping fashion described; and it is also logically possible that acoustic and semantic processing could be conducted in parallel.

The present experiment applies visual search techniques to the reading of continuous prose. There are three classes of target: visual, phonemic, and semantic. Search times for the single targets and for double and triple combinations of all classes are compared, and the temporal relations between these are used as a basis for inferring whether the analyses that are performed during reading occur in series, in parallel, or in overlapping sequence.

\section{Materials \\ METHOD \\ Passages of English prose were} constructed in such a way that all were 100 words in length, and each contained 10 targets. Seven types of passage were prepared. These were: (1) Visual target (V)-a specified letter. (2) Acoustic target (A)-a specified phoneme. (3) Semantic target (S)-words of a specified class. (4) Visual and acoustic targets $(\mathrm{V}+\mathrm{A})$-targets of both types occurring in the passage. (5) Visual and semantic targets $(V+S)$-both types occurring. (6) Acoustic and semantic targets $(A+S)$-both types occurring. (7) Visual, acoustic, and semantic targets $(V+A+S)-a l l$ three types of target occurring in the passage.

The passages were assembled into two groups. In the first group there were three passages of each of the seven types listed above. Different targets were assigned in each of these 21 passages. In the second group there were again 21 passages, three of each type. The same targets were reassigned randomly so that those that occurred as single targets in Group 1 occurred in the double or triple combinations in Group 2. This was done in case targets of unequal difficulty might bias the scores in some conditions. Targets were chosen for being well defined and easily discriminable, although choice was to some extent constrained by the need to keep constant the number of targets occurring within passages of a fixed length while conforming to normal prose style, i.e., those that tend to occur more or less frequently than 10 times per 100 words could not be used. The targets used were as follows:

Visual targets. These were all single letters occurring in their lower-case forms. The letters were $\mathrm{u} \mathrm{p} \mathrm{m} \mathrm{f} \mathrm{g} \mathrm{v} \mathrm{c} \mathrm{y} \mathrm{j} \mathrm{k} \mathrm{w} \mathrm{d.}$

Acoustic targets. These were all vowels or diphthongs produced by either single letters or clusters. For each target phoneme, different spellings were possible so that the target could not be detected visually. Ss were told that spelling would not delineate a target invariably and were given some representative examples to make this clear. In no case was semantic disambiguation necessary to distinguish a target, as, for example, in "lead" and "lead." The phonemes used were (IPA notation) el as in late, ov as in bone, $\partial \mathrm{I}$ as in like, $i$ as in feet, $\supset$ as in law, $u$ as in boot, av as in cow, $\varepsilon_{\mathrm{r}}$ as in hair, $a$ as in hot, $\mathrm{OI}$ as in toy, $v$ as in look, $\wedge$ as in but.

Semantic targets. These were chosen to be as familiar and unambiguous as possible. It was not feasible to keep the size of the sets constant. The classes were animals, flowers, furniture, colors, tools, fruit, clothing, vehicles, parts of the body, toys, professions, kitchen utensils.

Two targets never occurred together in the same word, since it lies outside the scope of this experiment to investigate any Stroop-like conflicts that might occur. The passages were typed on separate sheets of paper.

\section{Procedure}

Twelve subjects were employed. They were male and female paid volunteers, either graduates or undergraduates, without previous practice in search tasks. Six were assigned at random to the passages in Group 1 and six to Group 2.

The order of presentation for the 21 passages in each group was balanced to equate practice effects. The subject was handed the first sheet face downwards. He was told which target or targets to search for in that passage. Several examples of the target were given, though not, of course, any that actually occurred in the passage. Target specification was repeated until the subject was satisfied he had memorized 
Table 1

\begin{tabular}{lrrrrrrr}
\hline & $\mathrm{S}$ & $\mathrm{V}$ & $\mathrm{A}$ & $\mathrm{V}+\mathrm{A}$ & $\mathrm{V}+\mathrm{S}$ & $\mathrm{A}+\mathrm{S}$ & $\mathrm{V}+\mathrm{A}+\mathrm{S}$ \\
\hline $\begin{array}{l}\text { Mean time per } \\
\text { target detected }\end{array}$ & 2.39 & 3.50 & 4.90 & 6.86 & 4.37 & 4.52 & 7.03 \\
$\begin{array}{l}\text { Estimated total time } \\
\text { without omissions }\end{array}$ & 23.9 & 35.0 & 49.0 & 68.6 & 43.7 & 45.2 & 70.3 \\
$\begin{array}{l}\text { Prediction on the } \\
\text { additive model }\end{array}$ & & & 72.0 & 46.9 & 60.9 & 83.9 \\
$\begin{array}{l}\text { Difference from the } \\
\text { additive prediction }\end{array}$ & & & -3.4 & -3.2 & -15.7 & -13.6 \\
$\begin{array}{l}\text { Prediction on the } \\
\text { parallel model } \\
\begin{array}{l}\text { Difference from the } \\
\text { parallel prediction }\end{array}\end{array}$ & & 49.0 & 35.0 & 49.0 & 49.0 \\
\hline
\end{tabular}

Table 2

Error Distribution Showing Percentages of Errors for All Ss. The errors are all omissions. The total number of targets occurring in each of the seven conditions was 360 .

\begin{tabular}{lccccccc} 
& $\mathrm{S}$ & $\mathrm{V}$ & $\mathrm{A}$ & $\mathrm{V}+\mathrm{A}$ & $\mathrm{V}+\mathrm{S}$ & $\mathrm{A}+\mathrm{S}$ & $\mathrm{V}+\mathrm{A}+\mathrm{S}$ \\
\hline Percent errors in each condition & .83 & 12.5 & 18.05 & 19.7 & 8.05 & 12.2 & 11.9 \\
Visual errors & - & 12.5 & - & 9.44 & 4.44 & - & 4.44 \\
Acoustic errors & - & - & 18.05 & 10.26 & - & 10.55 & 5.27 \\
Semantic errors & .83 & - & - & - & 3.61 & 1.66 & 2.22 \\
\hline
\end{tabular}

them. The E gave a "ready" signal, followed by a "start" signal. The subject was instructed to turn over the sheet and start reading the passage, canceling all examples of the target or targets as he located them, and to call out "finished" when he reached the end of the passage. He was told to go as fast as possible, to try to avoid errors, and not to backtrack. In the double and triple target searches, the subject was told to give equal attention to all targets, and to try to look for them simultaneously as he worked through the passage, i.e., not to look for one target and then backtrack to scan again for the second target. The time taken from the "start" signal to the finish was measured by stopwatch. Each subject worked through all 21 passages of the group to which he was assigned in a single session lasting approximately $30 \mathrm{~min}$.

\section{RESULTS}

Time and errors were scored for each passage. The total time was divided by the number of targets detected, to give an approximate estimate of the time to detect each target. This method of scoring was necessary because Ss detected varying numbers of targets, and their search time varied with accuracy. For each $S$, means were calculated for the three passages of each of the seven types. Group means were also computed for the seven conditions. The two groups were compared to see if any difference due to the different allocation of specific targets was discernible. Although Group 2 was slower throughout, the rank order of the seven conditions was the same for the two groups, and the differences between the conditions were of a similar order of magnitude. It was therefore considered legitimate to combine the data of both groups.

Analyses of variance were performed on the scores of each group separately and on the combined data. Analyses were two-way, Subjects by Conditions. All yielded significant $F$ values for the condition factor: Group 1, $F(6,5)=5.35$; Group 2, $F(6,5)=6.20$; combined data, $F(6,11)=6.85, \quad p$ for all $F s<.05$. A Newman-Keuls test was then performed for each analysis of variance to find which of the conditions differed significantly from which of the others. The three Newman-Keuls tests all yielded the same result, significant at $\mathrm{p}<.01 . \mathrm{S}<\mathrm{V}<\mathrm{A}=$ $\mathrm{A}+\mathrm{S}=\mathrm{V}+\mathrm{S}<\mathrm{V}+\mathrm{A}=\mathrm{V}+\mathrm{A}+\mathrm{S}$.

The error distributions are given in Table 2. Only the errors of omission were included, since errors of commission were very few. The proportions of errors occurring in the different conditions was very consistent across $\mathrm{Ss}$.

Predictions on additive (serial) and parallel models are shown in Table 1 . The predictions on the additive model are derived as follows: The average total time to complete a passage without omissions is estimated by multiplying the mean time per target detected by 10 , the maximum number of targets occurring. On an additive prediction, the times for multiple search should equal the sum of the times for the relevant single searches. However, when the single search times are summed, the scanning time and the response time are duplicated in the double searches and trebled in the triple search. A constant is therefore subtracted from the summed times. The constant, $12 \mathrm{sec}$, is estimated from the mean time taken to read the passages as rapidly as possible and make 10 cancellations at random. The extent to which the totals in the multiple-search conditions deviate from these predictions is given in the table. On the parallel prediction, scores for the multiple searches should equal the score for the longest of the relevant singles. The extent to which actual scores deviate from the parallel predictions are also given in the table.

\section{Times for Single Targets}

\section{DISCUSSION}

The differences between single targets of different types are not easy to evaluate. A number of factors probably contribute to these differences. The size of the target is an obvious one. Semantic targets are comprised by whole words, and processing in units of this size reduces the total number of decisions to be made. But size of unit alone cannot be crucial since the larger phonemic units take longer than the smaller letter targets. The phoneme targets may be harder to discriminate from nontargets, and the task of phoneme detection in written material is probably less practiced. The disparity between times for visual and for phonemic searches in the present experiment gives no support to the view of Corcoran and Weening (1968) that acoustic processing occurs during letter search. The difficulty of the phonemic search also makes it unlikely that reading normally proceeds by mapping phoneme to grapheme, as Gibson, Pick, Osser, and Hammond have suggested (1962). Normal reading habits would tend to favor semantic search. At the perceptual stage, the reader selects the most informative parts of the word, usually the first and last letters (Bruner \& O'Dowd, 1958), and constructs or synthesizes the whole word from these. Such a strategy would obviously be faster than letter search. In letter search, if the letters are synthesized on the basis of tests for distinctive features, the number of constructs or acts of synthesis would be far greater. The present results are at variance with those of Neisser and Beller (1965) since they found that, although search for a specific word (Monday) was faster than search for a letter (K), search for a word of a specific class (names of American states) was slower. However, in the present experiment the use of continuous prose, rather than columns of unrelated material, provides contextual cues and linguistic redundancy to assist semantic search. The rank order for single targets $\mathrm{S}<\mathrm{V}<\mathrm{A}$ was identical for every subject. 
Table 3

Additional Mean Time Per Target

Detected (in Seconds) for Each Combination of Targets

Adding $S$ to $V(V+S)$ adds .87

Adding $S$ to $A(A+S)$ adds -.38

Adding $\mathrm{S}$ to $\mathrm{V}+\mathrm{A}(\mathrm{V}+\mathrm{A}+\mathrm{S})$ adds .17

Adding $V$ to $A(V+A)$ adds 1.96

Adding $V$ to $S(V+S)$ adds 1.98

Adding $V$ to $A+S(V+A+S)$ adds 2.51

Adding $\mathrm{A}$ to $\mathrm{V}(\mathrm{V}+\mathrm{A})$ adds 3.36

Adding $A$ to $S(A+S)$ adds 2.13

Adding $A$ to $V+S(V+A+S)$ adds 2.66

\section{Times for Multiple Targets}

It is clear that the additional time taken for double targets is scarcely, if at all, determined by the increase in number of targets, since all the double targets do not show the same size of increase, and there is no increase at all in the $A+S$ condition over the $S$ condition. The increased time seems to result from the difficulty of combining two types of search, and some combinations are more difficult than others. Adding a target of a given type does not always produce the same effect. For example, the addition of a visual target does not add a constant amount to all other conditions, as Table 3 shows. The processing time results from the combination, not the number or the type of its elements.

\section{Comparison of Results with Predictions from the Models}

Some of the actual scores are quite close to the predictions from the models. Both the $\mathrm{V}+\mathrm{A}$ and the $\mathrm{V}+\mathrm{S}$ conditions yield times only a little less than the additive prediction. Since for any individual unit some visual processing must precede either an acoustic or a semantic decision the decisions would necessarily be sequential. However, with continuous prose, it is possible that the visual processing of the next unit could begin while the semantic or acoustic processing of the previous unit was still taking place. An overlapping sequence of this kind would give search times lying somewhere between the predictions from the serial and from the parallel models.

The $A+S$ condition is not significantly slower than the $A$ condition and conforms to the parallel prediction. The semantic target can be searched for in addition to the slow phonemic target without adding extra time. Similarly, if the $\mathrm{V}+\mathrm{A}+\mathrm{S}$ combination is compared to the $\mathrm{V}+\mathrm{A}$ condition, rather than $A$ alone, it is not significantly slower than $\mathrm{V}+\mathrm{A}$. It is clear that semantic search proceeds in parallel with the combined visual and acoustic search in this condition.

Clearly no single answer emerges. The analyses performed in reading do not all combine together in exactly the same way. Combination search tasks requiring analysis at the lowest level of the hierarchy, the visual stage, are carried out in a quasiserial or overlapping fashion. Combinations requiring analyses at the two upper levels of the hierarchy, the acoustic and the semantic, can be performed in parallel.

The failure to find a unitary model applicable to all combinations of analyses should not be surprising. When the analyses are of unequal difficulty, employ units of unequal size, and are at different hierarchical levels, it is not to be expected that they can be operated in conjunction with equal facility.

\section{REFERENCES}

BRUNER, J. S., \& O'DOWD, D. A note on the informativeness of parts of words. Language \& Speech, 1958, 1, 98-101.

COHEN, G. A comparison of semantic, acoustic and visual criteria for matching of word pairs. Perception \& Psychophysics, 1968, 4 203-204

CORCORAN, D. W., \& WEENING, D. L Acoustic factors in visual search. Quarterly Journal of Experimental Psychology, 1968, 20, 83-85.

GIBSON, E. J., PICK, A., OSSER, H., \& HAMMOND, M. The role of grapheme-phoneme correspondence in the perception of words. American Journal of Psychology, 1962, 75, 554-570.

KAPLAN, I. T., CARVELLAS, T., \& METLAY, W. Visual search and immediate memory. Journal of Experimental Psychology, 1966 , 71, 488-493.

NEISSER, U. Visual search. Scientific American, $1964,210,94-102$.

NEISSER, U., \& BELLER, H. K. Searching through word lists. British Journal of Psychology, 1965, 56, 349-358.

\section{NOTES}

1. This work was carried out while the author was in receipt of a Medical Research Council scholarship.

2. Address: Institute of Experimental Psychology, 1 South Parks Road, Oxford, England.

(Accepted for publication February 9, 1970.) 